Itinéraires Itinéraires

Littérature, textes, cultures

2011-1| 2011

Les Mémoires, une question de genre?

\title{
Madame de Boigne, entre écriture politique et émergence d'une sensibilité romanesque
}

\section{Henri Rossi}

\section{(2) OpenEdition}

1 Journals

Édition électronique

URL : http://journals.openedition.org/itineraires/1615

DOI : 10.4000/itineraires. 1615

ISSN : 2427-920X

Éditeur

Pléiade

\section{Édition imprimée}

Date de publication : 1 avril 2011

Pagination : 65-72

ISBN : 978-2-296-13692-2

ISSN : $2100-1340$

\section{Référence électronique}

Henri Rossi, « Madame de Boigne, entre écriture politique et émergence d'une sensibilité romanesque », Itinéraires [En ligne], 2011-1 | 2011, mis en ligne le 01 avril 2011, consulté le 25 juin 2020. URL http://journals.openedition.org/itineraires/1615; DOI : https://doi.org/10.4000/itineraires.1615

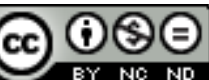

Itinéraires est mis à disposition selon les termes de la licence Creative Commons Attribution - Pas d'Utilisation Commerciale - Pas de Modification 4.0 International. 


\title{
Madame de Boigne, entre écriture politique et émergence d'une sensibilité romanesque
}

\begin{abstract}
The countess of Boigne belonged to an ancient but enlightened aristocracy. Her education, mainly provided by her father, marquis of Osmond, stimulated in her at a very early age a strong interest in philosophy, but also and mainly in politics. The marquis of Osmond happened to be appointed as King Louis XVIII's ambassador in Turin and London. Madame de Boigne was also a very close friend of the Orleans family. Thus, she was frequently to be found in the immediate entourage of powerful people. However, while putting to paper her experience and analysis of political events of her time, the countess of Boigne never uses the cold and impersonal style of an impartial witness. Ideological and political reflections are not determined by any purely intellectual abstraction but rather by female sensitivity.
\end{abstract}

Keywords : memoirs, autobiography, Restauration, sensitivity, woman's writing Mots clés : Mémoires, autobiographie, Restauration, sensibilité, écriture féminine

Adélaïde d'Osmond, née en 1781, morte en 1866, a laissé de volumineux Mémoires consacrés à la période allant de la Révolution française à la fin de la monarchie de Juillet. Comme la plupart des récits mémoriels écrits et publiés par les femmes de l'aristocratie à la suite de la Révolution, ils nous confrontent au problème maintes fois soulevé par tous ceux qui s'intéressent aux Mémoires issus de la Révolution, de George Gusdorf à Damien Zanone, à savoir la ligne de démarcation - si l'on veut prendre une métaphore géographique -, l'équilibrage - si on l'emprunte à la physique -, qui peuvent exister entre l'écriture personnelle, la part autobiographique, et l'effacement du moi, la mise à distance de soi-même.

On retrouvera chez Madame de Boigne, et je n'insisterai pas sur ce point car il a été de nombreuses fois soulevé et étudié, la revendication d'une écriture aristocratique, et le refus affiché de donner à ses Mémoires la coloration d'une autobiographie. Les formules sont nombreuses qui apparentent 
les Récits d'une tante (ainsi la comtesse de Boigne nomme-t-elle ses écrits, dédiés à ses neveux) aux Mémoires aristocratiques les plus sourcilleux : « revoir [la copie], c'est tout à fait inutile; je ne sais pas écrire, à mon âge, je n'apprendrai pas le métier... »; « J'ai parlé de moi, trop peut-être, certainement plus que je ne l'aurais voulu... ${ }^{2} »$, etc., répète-t-elle à l'envi.

La revendication la plus nette émise par la mémorialiste est de donner à ses Mémoires la coloration de Mémoires politiques. Elle annonce clairement son intention dès l'avant-propos de son récit, intitulé, avec un humour et une ironie propitiatoires, «Au lecteur, s'il y en $\mathrm{a}^{3}$ » :

[...] peut-être sera-t-il assez curieux d'observer comment, dans [les temps] où j'ai vécu, la force des circonstances m'a toujours entraînée à être une personne de parti, tandis que, par instinct, par goût et par raisonnement, j'avais horreur de l'esprit de parti et que je jugeais assez sainement des fautes et des ridicules où il conduit ${ }^{4}$.

Le ton est ainsi donné dès le début des Mémoires : ils seront plus l'histoire d'une personne confrontée aux événements politiques, et de la part qu'elle a pu y prendre, que celle d'événements personnels. Au chapitre II de la quatrième partie, elle fait l'éloge de la politique et de l'analyse comme compensation à la religion :

Je trouve que lorsqu'on n'est pas assez heureusement organisé pour s'occuper exclusivement et religieusement du sort futur qui doit nous être éternel, ce qu'il y a de plus digne d'intérêt pour un esprit sérieux, c'est l'état actuel des nations sur la terre ${ }^{5}$.

Et il est de fait que la comtesse de Boigne est remarquablement placée pour nous livrer, fût-ce avec le décalage du temps, ses récits d'événements, ses analyses politiques. Fille du marquis d'Osmond, elle vit à Versailles les événements de la Révolution, dans les entours de la famille royale; sous la Restauration, son père ayant été nommé ambassadeur, successivement à Turin et à Londres, elle fait les honneurs des salons de l'ambassade et se trouve ainsi en première position pour observer, consigner, analyser ce qu'elle nomme elle-même «le dessous des cartes ». Ayant noué avec le chancelier Étienne-Denis Pasquier une amitié amoureuse qui les unira jusqu'à la mort du chancelier en 1862, elle est également une intime de la famille d'Orléans, amie proche de Marie-Amélie, et elle fréquente

1. Les Récits d'une tante ont été publiés pour la première fois à Paris, Plon et Nourrit, 19071908; réédités à de nombreuses reprises : Paris, Émile-Paul, 1921-1925; Paris, Mercure de France, coll. «Le temps retrouvé », éd. Berchet, 1971, 1979, 1986 et 1999; Paris, Honoré Champion, éd. Rossi, 2007.

2. Adèle d'Osmond de Boigne, Récits d'une tante, Paris, Honoré Champion, 2007, p. 48.

3. Ibid., p. 47.

4. Ibid., p. 48.

5. Ibid., p. 388. 
assidûment les cercles du pouvoir sous la monarchie de Juillet... Elle joue même un rôle, officieux mais non négligeable, au moment des Trois Glorieuses, ayant contribué à l'accession au trône du roi des Français.

Ce qu'il y a de remarquable est que cette écriture de l'analyse politique va de pair avec une largeur de vues sur les événements en cours, qui apparente à certains moments les Récits d'une tante aux Considérations sur la Révolution française de Madame de Staël. La comtesse de Boigne, loin d'être réactionnaire et de vouloir faire agir, au moment de la Restauration, ce que Jean-Louis Bory appelait « la machine à remonter le temps », (ce que ses origines pouvaient nous laisser croire et craindre), s'inscrit dans le sens de l'Histoire et restitue cette ouverture dans une écriture analytique, parfois froide et sèche, allant jusqu'à écrire : " C'est ainsi que la Révolution nous a faits ${ }^{6} . »$ Dès son plus jeune âge, elle est rompue à l'analyse économique et la philosophie politique ne lui est pas étrangère : durant son émigration en Angleterre, elle lit, à l'âge de 15 ans et en manière de récréation, l'ouvrage d'Adam Smith, Recherches sur la nature et les causes de la richesse des nations, publié en 1776, à la grande surprise de Calonne, ce qui lui fait écrire : « les rires de monsieur de Calonne [...] furent pour moi le premier avertissement que ce goût n'était pas général aux filles de quinze $a_{n}{ }^{7}$ ».

Dès l'âge de 19 ans, en voyage à Munich, elle se forge la conception politique qui sera la sienne tout au long de sa vie : «j'y trouvai l'exagération poussée à un point d'extravagance qui me confondit [...]. Je m'accoutumai dès lors à n'être de l'avis de personne et inventai le juste milieu à mon usage ${ }^{8} . »$

Alors évidemment, nous sommes ici dans l'écriture rétrospective et tout le problème est de savoir, nous interrogeant sur Mémoires et vérité, s'il fut vrai que la jeune Adèle, dès son plus jeune âge, se montrât ainsi un puits d'intellectualisme et de réflexion sur les événements en cours. Les Mémoires sont aussi recherche d'une vérité de soi, certes, mais quête d'un sens global de l'existence, recherche d'une cohérence, ce que Gusdorf, dans les Ecritures du moi, nommait, « la recherche d'un centre ${ }^{9} »$. Madame de Boigne, dès les premiers mots de son récit, s'attache à reconstituer l'unité et la cohérence d'une pensée politique qui connaît son plein achèvement au moment de l'accession au trône de Louis-Philippe en 1830. Car la cohérence de son existence, elle ne peut précisément la trouver que dans son évolution politique, sa vie personnelle ayant été plutôt décevante. Lorsqu'elle écrit, priant son lecteur de l'excuser d'avoir parfois envahi son récit par l'usage

6. Ibid., p. 421.

7. Ibid., p. 181-182.

8. Ibid., p. 210.

9. George Gusdorf, « De l'autobiographie initiatique à l'autobiographie genre littéraire », Revue d'Histoire Littéraire de la France, n 75/6, p. 957-994, nov.-déc. 1975, p. 972. 
d'un « je » par trop omniprésent - « Il a fallu que ma vie servît comme de fil à mes discours ${ }^{10} »-c^{\prime}$ est le fil de sa pensée politique qu'elle évoque :

Il serait assurément fort peu intéressant de connaître mes opinions personnelles en 1814. Mais c'est une recherche qui m'amuse de me rendre ainsi compte de moi-même aux différentes époques de ma vie et d'observer les variations qui les ont marquées ${ }^{11}$.

Mais la comtesse va aussi échapper à une écriture strictement analytique et distanciée en mettant sa personne, sa sensibilité en cohérence parfaite avec son évolution politique. Alors qu'elle se revendique comme un esprit sérieux, elle n'échappe pas, soit en toute conscience, soit peut-être piégée par son inconscient, à une écriture où politique et sensibilité se mêlent harmonieusement, la «variation »d'opinion étant étroitement liée à l'émotion éprouvée.

L'exemple le plus probant de cet enchevêtrement entre sensibilité et politique est l'épisode de l'éxécution de Charles de La Bédoyère à propos duquel la comtesse se revendique à la fois comme détentrice d'une pensée politique et porteuse d'une réflexion sur l'événement, et comme femme, son sexe déterminant sa prise de position.

Charles de La Bédoyère, qui combattit sous les bannières impériales et vouait à l'empereur Napoléon une admiration sans bornes, se laissa gagner à la cause des Bourbons pour plaire à sa belle-famille, éminemment légitimiste, les Chastellux. Mais, en 1814, lorsque l'empereur revient de l'île d'Elbe et semble voler vers la victoire, La Bédoyère qui, au fond, n'a jamais abandonné ses idées bonapartistes, se rallie à Napoléon et le rejoint à Vizille. Il est nommé comte d'Empire, promu général de brigade. Après la défaite de Waterloo, il est condamné à mort et exécuté le 19 août 1814. Madame de Boigne revient du Piémont, de Turin, son père ayant eu sa nouvelle affectation pour Londres. Elle fait étape à Lyon et c'est là qu'elle apprend l'exécution de La Bédoyère, par les journaux qu'elle trouve à l'hôtel de l'Europe.

J'y lus la condamnation de monsieur de La Bédoyère et j'éprouvais un mouvement d'horrible joie. «Enfin, me dis-je, voilà un de ces misérables traîtres puni! »Ce mouvement ne fut que passager; je me fis promptement horreur à moi-même; mais enfin, il a été assez positif pour avoir pesé sur ma conscience. C'est depuis ce moment, depuis le dégoût et le remords qu'il m'inspire, que j'ai abjuré, autant qu'il dépend de moi, les passions de l'esprit de parti et surtout ses vengeances. Je pourrais, à la rigueur, me chercher une excuse dans tout ce que je venais d'apprendre à Chambéry sur la conduite de monsieur de La Bédoyère, dans les tristes résultats que sa coupable trahison avait attirés, dans l'aspect de la patrie déchirée et envahie par un million d'étrangers; mais rien n'excuse, dans un cœur féminin, la

10. Adèle d'Osmond de Boigne, op. cit., p. 48.

11. Ibid., p. 371. 
pensée d'une sanglante vengeance, et il faut en renvoyer l'horreur à qui il appartient, à l'esprit de parti, monstre dont on ne peut trop repousser les approches quand on vit dans un temps de révolution et qu'on veut conserver quelque chose d'humain ${ }^{12}$.

Madame de Boigne se réclame ici d'une sensibilité toute féminine, en même temps que d'une réflexion politique soigneusement documentée, mais ce qui parait intéressant, c'est que la sensibilité féminine ici revendiquée, s'opposant à la passion générée par l'esprit de parti et jouant le rôle d'un régulateur est, en définitive, le vecteur d'une pensée plus juste, plus humaine, mais aussi plus réfléchie... La sensibilité dont elle se réclame et qui provoque cette espèce de conversion définitive à une école de la modération, loin de perturber ou de dévoyer la pensée politique, loin de susciter un mouvement spontané, irréfléchi et peut-être porteur de contre-vérité, lui donne une assise plus stable, plus durable. Sensibilité et réflexion se marient pour créer une forme de vérité idéologique absolue à laquelle elle prétend atteindre.

Encore est-ce ici en toute conscience que Madame de Boigne se revendique comme femme, ce qu'elle attribue comme composante d'un cœur féminin, la sensibilité, générant une réflexion politique plus pertinente.

Mais il va lui arriver de se laisser piéger par son inconscient (à moins que, ici encore, elle agisse en toute conscience?) pour nous proposer, à travers un récit enchâssé, une sorte de mini-roman vécu qu'elle insère dans son propos, pour nous livrer, en filigrane, comme en un message subliminal, tout le drame de sa vie, qu'elle se refuse à traiter comme tel dans le cours de ses Mémoires. Un « esprit sérieux », pour reprendre son expression, ne se laisse pas aller ainsi à dilater l'état de son âme à propos de faits personnels et intimes. Pourtant cet état va apparaitre, à travers des transformations, plus ou moins conscientes, infligées à la vérité chronologique, de façon que l'écriture provoque une mise en parallèle implicite de l'événement qu'elle raconte et du drame de son existence.

Madame de Boigne reste globalement fort discrète sur l'événement douloureux qui marque toute sa vie : son mariage avec le général Bennett de Boigne, richissime mercenaire au service de l'Angleterre, tout d'abord, d'un prince indien ensuite, toutes activités qui lui permettent de bâtir une colossale fortune.

Alors que les Osmond, émigrés à Londres, vivent dans la misère la plus complète, le général de Boigne, fraîchement retraité, s'éprend de la jeune Adèle, lui propose sa main, qu'elle accepte pour sortir sa famille de la détresse. Elle épouse le nabab le 8 juin 1798. Le mariage se révèle vite une catastrophe (le général a 30 ans de plus que sa femme, il souffre de troubles nerveux, la tyrannise, deux séparations ont lieu assez rapidement après la noce). 
Après avoir raconté, d'une manière très générale, quelques épisodes douloureux de cette triste union, elle affirme : « Je n'entrerai plus dans aucun détail sur mon mariage ${ }^{13} \gg$. Trois pages plus loin, elle renouvelle cette affirmation: «Si je ne m'étais promis de ne plus entrer dans ces détails, j'aurais un long récit à faire de tout ce que les mauvaises façons de monsieur de Boigne me firent souffrir ${ }^{14}$. »

Or, juste après cela, elle va insérer dans son récit, après nous avoir également livré son analyse politique des excès de l'émigration à Coblence et avoir affirmé son attachement idéologique au « juste milieu », un roman enchâssé, celui de son amie Mary Kingston et de la passion amoureuse dévorante que cette jeune fille a connue avec son cousin, le colonel Henry Fitzgerald.

Tous les éléments romanesques sont là, empruntés à la littérature sentimentale du XVIII ${ }^{\mathrm{e}}$ en même temps qu'elle émaille son récit de notations dignes de la Princesse de Clèves :

Lady Mary était une des personnes les plus charmantes que j'aie jamais rencontrées [...]. Quand on aura compulsé tous les portraits de héros de roman pour en extraire l'idéal de la perfection, on sera encore au-dessous de ce qu'il y aurait à dire du colonel Fitz-Gerald. Sa belle figure, sa noble tournure, sa physionomie si douce et si expressive n'étaient que l'annonce de tout ce que son âme contenait de qualités admirables ${ }^{15}$.

Le colonel Fitzgerald, comme tout héros de roman, chante à ravir, la voix de lady Mary se mêlant à la sienne. Fitzgerald, marié, abandonne sa femme et enlève Mary : son châle et son chapeau, retrouvés au bord de la rivière, laissent croire, dans une tradition romanesque qui a fait ses preuves, à un suicide. En réalité, Mary s'est enfuie avec Fitzgerald. La famille de Mary la récupère, elle est enceinte des œuvres du colonel. Le père et les frères de lady Mary traquent le colonel, l'attirent dans un piège et l'assassinent dans la nuit du 11 décembre 1797.

Ce qu'il y a d'intéressant ici est, bien entendu, le fait que le genre mémorialiste se trouve contaminé par le genre romanesque; mais c'est là une tradition des Mémoires, depuis le cardinal de Retz, que de donner à des épisodes réels de l'existence les couleurs du romanesque et l'amplification propre au genre.

Mais ce qui me paraît plus significatif est le fait que, consciemment ou non, la comtesse de Boigne se laisse progressivement aller à un travestissement de la chronologie afin de mettre en parallèle le destin amoureux, si parfaitement réussi sur le plan sentimental, de son amie Mary Kingston, et le sien propre, si tragique et si porteur de désillusion. Comme si, en

13. Adèle d'Osmond de Boigne, op. cit., p. 209.

14. Ibid., p. 211.

15. Ibid., p. 211. 
lisant le roman de Mary, lequel connaît certes une fin épouvantable mais offre aussi une plénitude affective, devait se révéler, aux yeux du lecteur, le roman désespérant, et, hélas, durable, que Madame de Boigne est en train de vivre.

Le roman de Mary Kingston et de Fitzgerald débute en effet dans les premiers mois de l'année 1797 (Mary était née en 1780 et la comtesse nous dit que, au moment où s'ébauche l'idylle avec le colonel, elle « atteignait sa dix-septième année »). L'idylle commence d'ailleurs sous les yeux de la comtesse, le colonel servant en quelque sorte de chaperon à sa cousine Mary. Avant qu'il soit déclaré pleinement, Madame de Boigne s'associe à cet amour naissant : " Nous faisions chanter [le colonel] avec nous, et c'étaient des joies et des rires lorsqu'il manquait un dièse ou estropiait une syllabe italienne ».

Durant l'été suivant, en 1797, Fitzgerald est chargé d'amener sa cousine en Irlande, afin qu'elle y rejoigne sa mère : «C'est dans ce fatal voyage, écrit la comtesse, qu'ils succombèrent tous deux à la passion qui les dominait. »

Or, Adèle, qui est toujours à Londres à ce moment-là, va opérer une transformation de dates afin de faire coïncider, en miroir, en négatif en quelque sorte, l'histoire passionnelle de lady Mary et son mariage d'argent. « Mon mariage, écrit-elle, eut lieu pendant cette absence. » Or nous savons que Madame de Boigne ne connaît pas encore son mari durant l'été 1797, elle ne le rencontrera qu'au mois de mai de l'année 1798.

Elle va dès lors travestir les dates (sans d'ailleurs les donner précisément, mais la généalogie britannique est précise quant aux datations des familles Kingston et Fitzgerald), ce qui lui permet de ne plus être un témoin direct des événements, mais d'en avoir uniquement des échos, soit par les lettres que Mary est supposée lui avoir envoyées, soit par les dires de sa mère, la marquise d'Osmond.

En effet, durant l'automne 1797, alors que, de retour à Londres, Mary se laisse aller à sa passion pour le colonel Fitzgerald, alors que s'organisent l'enlèvement de la jeune fille, sa récupération par sa famille, Madame de Boigne écrit : «J'étais sur le continent lorsque les alarmes que donnaient son dépérissement et sa profonde tristesse ${ }^{16} \ldots$ » Or, le voyage que la comtesse effectue avec son mari en Europe continentale a lieu en 1800, soit plus de deux ans et demi après ces événements. Mais il importe à Madame de Boigne, et cette écriture romanesque, génératrice d'une écriture du moi qui se masque sous un récit tiers, obéit-elle à sa conscience ou s'agit-il ici d'un simple défaut de la mémoire?, de faire coïncider le destin passionnel de Mary et de Fitzgerald avec le sien, dont le lecteur ne connaît pas les détails (mais elle a eu soin tout de même, quelques pages plus haut, de

16. Le roman de Mary Kingston est raconté p. 211-216. 
nous dire que son mariage fut malheureux et que le voyage en Europe avec Boigne ne fut pas une réussite), mais dont il suppose toute la violence conjugale au quotidien. La mort de Fitzgerald intervenant le 11 décembre 1797, on est évidemment loin du mariage de Madame de Boigne, et encore davantage du voyage en Europe.

La transformation des dates et leur mise en corrélation renvoient l'écriture des Récits d'une tante, à de certains moments du moins, à une écriture qui se revendique comme hautement féminine, non dans sa forme (on retrouve dans ce mini-roman des accents rousseauistes), mais dans sa thématique, usant d'un procédé romanesque, la duplication, faisant de Mary un double rêvé.

Henri Rossi

Université de Picardie Jules Verne 